\title{
Non-contact experimental assessment of apparent dynamic stiffness of constrained-layer damping sandwich plates in a broad frequency range using a Nd:YAG pump laser and a laser Doppler vibrometer.
}

\author{
N.B. Roozen ${ }^{1, *}$, L. Labelle ${ }^{1}$, Q. Leclère $^{b}$, K. Ege ${ }^{\mathrm{b}}$, S. Alvarado $^{1}$ \\ ${ }^{a}$ Katholieke Universiteit Leuven, Laboratory of Acoustics, Soft Matter and Biophysics, \\ Department of Physics and Astronomy, Celestijnenlaan 200D 3001 Leuven, Belgium. \\ ${ }^{b}$ Univ Lyon, INSA-Lyon, LVA EA677, F-69621, Villeurbanne, France.
}

\begin{abstract}
This paper concerns the assessment of the dynamic stiffness of plates using non-contact excitation and non-contact measurements. In particular, a constrained-layer damping sandwich plate is considered, of which the apparent dynamic stiffness as function of frequency is determined. The experimental results compare very well with an analytical model that computes the frequency dependent apparent bending stiffness of the constrained-layer damping sandwich plate. A Nd:YAG laser is used for excitation of the plate and a laser Doppler vibrometer is used to measure its dynamic response along a line on the plate, thus reducing the measurement effort. Using advanced data acquisition and data processing techniques, a signal-to-noise ratio of up to $100 \mathrm{~dB}$ was obtained, yielding estimates of the flexural wavenumber and dynamic stiffness of the plate up to a frequency of $50 \mathrm{kHz}$. It is shown that the Prony method and the wavenumber fit method yield a much improved wavenumber resolution as compared to the spatial Fourier transform method. An additional advantage of the wavenumber fit approach is that it allows the accuracy of the fit to be determined. The accuracy was estimated at $1 \mathrm{rad} / \mathrm{m}$ (best relative wavenumber resolution $2 \%$ ).
\end{abstract}

Keywords: Laser Doppler vibrometer, Nd-YAG laser, non-contact excitation, structural dynamics, dispersion, equivalent homogeneous plate,

${ }^{*}$ Corresponding author 
constrained-layer damping sandwich plate

PACS: 46.40.-f, 46.40.Cd, 46.80.+j

\section{Introduction}

In a constrained-layer damping sandwich structure two thin faces of metal sheet are bonded to a visco-elastic core. It provides an effective way to suppress vibration and noise in structures, whilst the structure can remain to be relatively thin and light, as compared to non-constrained damping treatments. This makes constrained-layer damping sandwich structures interesting for automotive, aerospace as well as naval applications.

For the analysis of constrained-layer damping sandwich structure onedimensional finite-element models have been developed to describe the crosssectional deformation of a linear viscoelastic laminate[1], as well as special '2.5D' layered shell elements (see for instance [2]). Other approaches can be used for the modeling of composite panels, based on the Wave Finite Element Method (WFEM) $([3,4])$ or analytic approaches $([5,6])$.

For the validation of these models it is required to perform measurements on constrained-layer damping sandwich test panels. This paper is dealing with the experimental assessment of the effective (complex) bending stiffness of test panels in general, and constrained-layer damping sandwich test panels in particular. It should also be noted that the apparent stiffness of constrained-layer damping sandwich plates are frequency dependent (see e.g. [7, 8]).

With respect to the measurement approach, in general one can distinguish contact and non-contact methods to excite the plate. Contact methods to excite a structure usually result in measurement data with a good signalto-noise ratio. Shakers are capable of injecting relatively high energy levels, resulting in vibration levels well above the noise floor. Mounting a shaker, however, is not always easy and requires a highly skilled experimenter. A good alternative is hammer excitation. A disadvantage of hammer excitation is that it can be rather difficult to repeatedly excite at the same physical location on the structure, causing so-called DOF-jitter [9].

Although for non-contact methods a highly skilled experimenter is required as well, and additional safety precautions are also necessary (laser beam), non-contact methods have the advantage that the structure remains untouched. This can be important in case of fragile structures, or in biomedical applications where the testing equipment must be sterile. Non-contact 
excitation can be accomplished by means of an electro-magnetic force [10] or an air pulse [11], among others. The electro-magnetic excitation employs the interaction between the eddy currents and the magnetic field to create a Lorentz force that excites the structure. Hefner et al. [10] examined the use of electromagnetic excitation of a stainless steel shell immersed in water. They found that excitation by means of electromagnetic forces are more suited to excite torsional modes. Hefner et al. excited a structure immersed in water up to frequencies as high as $100 \mathrm{kHz}$. In a modal analysis application Bau et al. 12] used this method of excitation to excite a structure immersed in air up to frequencies of $2000 \mathrm{~Hz}$. Obviously this excitation approach can only be used when the structure is made from an electrically conducting material.

Excitation by means of a pulsed air jet is an alternative way of noncontact excitation. This approach typically excites the structure in the lower frequency range, up to frequencies of approximately $200 \mathrm{~Hz}$ (e.g. [11, 13]), which is appropriate for experimental modal analysis, for instance.

Yet another way of non-contact excitation is by means of a laser impulse. This method of excitation is based on the photo-acoustic effect. The laser emits light at a very high intensity during an extremely short time (for instance, in the order of 1 nano second, depending upon the type of laser), focused at a point on the structure. Each light pulse heats up the material at the light spot, which locally expands the material and launches a vibrational wave. The material can be excited up to very high frequencies (e.g. $\mathrm{GHz}$ range) with this excitation technique.

Castellini [14] used a Nd:YAG source (532 nm, $100 \mathrm{~mJ} /$ pulse) to excite a beam structure and perform modal analysis on it up to frequencies of about $3000 \mathrm{~Hz}$. They also measured the structural response of the beam by means of a laser Doppler vibrometer, thus using an all-optical, non-contact approach, with obvious advantages.

In this paper it is shown that photo-acoustic excitation by means of a laser impulse (Nd:YAG-laser, wavelength $1064 \mathrm{~nm}$, maximum energy 200 mJ per pulse) can excite a constrained-layer damping sandwich structure (total thickness $1.17 \mathrm{~mm}$ ) at sufficiently high vibration levels, measurable by means of a laser Doppler vibrometer, up to frequencies as high as $50 \mathrm{kHz}$. Excitation at higher frequencies appeared for this specific plate structure not possible due to inertia effects of the plate and the limited excitation force of the Nd:YAG laser pulses. Once the laser beam is positioned at a single point on the plate, it remains accurately exciting this specific point on the plate. Because of this, time-averaging can be exploited to improve the signal-to- 
noise ratio, without the above mentioned DOF-jitter effects. Using a laser Doppler vibrometer to measure the response of the structure a signal-to-noise ratio of up to $100 \mathrm{~dB}$ was obtained. In this way, an all-optical, non-contact measurement approach for both excitation and response measurement was employed.

The data processing aspects that are addressed in this paper are mainly related to the conversion of the measurement data to dispersion relationships and the assessment of the dynamic stiffness of the plate. The estimation of the dispersion curves requires a transformation of the measurement data from the time-space domain to the frequency-wavenumber domain. Whilst the transformation from the time-domain to the frequency domain can usually be done with a sufficient frequency resolution by means of a classical Fourier transform, the transformation from the spatial domain to the wavenumber domain often suffers from a rather limited wavenumber resolution due to the limited scanning length along the sample. The article discusses alternative ways of the classical Fourier transform to determine the wavenumber from the measurement data in an effort to overcome this limitation, i.e. the Prony approach, and the wave fitting approach.

The Prony method, developed in the $18^{\text {th }}$ century, is a well-know approach in which damped sinusoids are used to fit equally spaced measurement data (for a contemporary treatment of modern Prony methodsm, see [15]). Many papers use this approach. For instance Braun and Ram [16] used the Prony approach for modal analysis and Grosh and Williams [17] used this method to estimate the complex wave-number of structural vibrations.

Yet another, closely related approach, is a wave fitting approach, which is in literature sometimes also called the inhomogeneous wave correlation method [18, 19, 20], the maximum likelihood method [21], the least-squares estimation method [22], or the wave coefficients method [23]. Basically, the method searches for (assumed) wave functions that fit the measurement data best in a least squares sense. Because the approach employs a redundancy of measurement data, the estimates of the parameters are robust. Moreover, as opposed to the Prony method, the wave fitting approach can also be applied to measurement data that was taken on non-equally spaced positions and the method allows for an estimation of the fit accuracy.

Halliday et al. [21], Hillstrom et al. [22], and also Liao et al. 23] applied the wave fitting approach to a beam structure. Hull et al. [24] used the method to estimate the complex flexural wavenumber of a beam. Rak et al. [20] applied it to estimate the loss factor of beams. 
Halkyard [19] studied the wave propagation in a plate with this method. Berthaut et al. [18] applied the wave fitting approach in two dimensions for the estimation of the dispersion curve of a ribbed panel, as function of the angle of propagation in the panel.

This paper focuses on the measurement of the apparent stiffness of constrainedlayer damping sandwich panels, in which the sandwich panel is represented by an equivalent Kirchhoff-Love homogeneous plate, as well as a comparison with an analytical model. Measurements are done in a contactless manner, using a Nd:YAG pump laser to excite the structure by photoacoustic effects, and a laser Doppler vibrometer to measure the response of the plate.

The paper employs both a laser for exciting the plate structure and sensing the vibrational response. Although the use of a pump laser for excitation of the structure is not new (see Castellini [14]), the device is scarcely used in the structural-dynamics community. This paper hopes to get more researchers and engineers interested in this approach.

Next to that the paper shows that the wavenumber fit approach can also be used to assess the accuracy of the plate stiffness as function of frequency, something which is not possible when using other approaches (such as the classical spatial Fourier transform). Furthermore, to the knowledge of the authors, the extraction of the stiffness parameters of a plate was never pursued on the basis of measurement data that was taken along a line on the plate. Measuring on a line, instead of an area of the plate, reduces the measurement effort. It is shown that the estimation of the wavenumber propagating in the plate can be done on the basis of a line-measurement, without compromising the quality of the material assessment.

On the modeling part, it is shown that the apparent stiffness (Young's modulus) of constrained-layer damping sandwich panels as predicted by an analytical model from the literature, using nominal values of the thickness and material properties of the individual layers of the sandwich plate, corresponds very well with the measured stiffness as function of frequency.

The paper is organized as follows. Section 2 discusses the three different data processing approaches to transform the measurement data from the spatial domain to the wavenumber domain. The test set-up that was used to apply these theories for the estimation of the material properties of a constrained-layer damping sandwich plate is detailed in section 3 . The results are discussed in section 4 , where in subsection 4.1 the results obtained with the standard spatial Fourier are shown and in subsections 4.2 and 4.3 the results obtained with the Prony approach and the wave fitting approach, 
respectively, are shown. The concluding remarks of the paper are given in section 5 .

\section{Data processing approaches}

In this section the theoretical basis is given for the estimation of the material properties of a plate-like structure from measurement data. It is the main objective of this paper to estimate the stiffness of the plate structure as function of frequency. The Young's modulus of an equivalent, homogeneous thin plate following Kirchhoff-Love plate theory 25] that represents the plate under investigation as good as possible, is examined.

It is assumed that the measurements are taken along a line on the plate. These points have coordinate $x$. The point of excitation is also chosen to be located on this line. Using this approach reduces the measurement efforts.

Consider measurement data of the form $w(x, t)$ where $w$ is the structural displacement in normal direction of the plate as function of coordinate $x$ and time $t$. The measurement data in the spatial-time domain, $w(x, t)$, are transformed to the spatial-frequency domain $w(x, \omega)$ by means of Fourier transform, i.e.

$$
w(x, \omega)=\mathcal{F}\{w(x, t)\}
$$

where $\omega$ is the angular frequency and where $\mathcal{F}$ denotes the Fourier transform. The transformation to the wavenumber-frequency domain $w(k, \omega)$, where $k$ is the wavenumber, can be done in different ways. In this section three methods are discussed: the classical spatial Fourier transform, the Prony method and the wave fitting approach.

The transformation of $w(x, \omega)$ to $w(k, \omega)$ by means of the classical spatial Fourier transform is denoted by

$$
w(k, \omega)=\mathcal{F}\{w(x, \omega)\}
$$

Prony analysis is a method of fitting a linear combination of exponential terms to a finite number of samples of a signal [15, 16, 17, 26]. The phase variation of the pole as function of the spatial coordinate $x$ gives the wavenumber $k$ :

$$
k(\omega)=\frac{\angle p(\omega)}{\Delta x}
$$

where $p$ is the Prony pole found at angular frequency $\omega$ and $\Delta x$ is the step size of the measurement points along $x$. 
The third and last approach that will be discussed in this paper is a wave fitting procedure, in which the $x$-dependence of the signal is fitted by a testwave for each angular frequency $\omega$. The method assumes, in it's most simple form, only one test-wave which is propagating through the structure. This test-wave $\phi$ is assumed to be a harmonic function of the following form

$$
\phi_{k}(x)=\frac{\mathrm{e}^{i k x}}{\sqrt{\left|x-x_{0}\right|}}
$$

where the suffix ${ }_{k}$ indicates the dependence on the (assumed) testing wavenumber $k, i$ is the imaginary number, and the term $1 / \sqrt{\left|x-x_{0}\right|}$ accounts for the geometric attenuation of the structural vibration level in an infinite plate as function of the distance from the point of excitation, $x=x_{0}$. In general, $k$ can be complex. However, in section 4 of this paper, only real values for $k$ will be considered as the plate under consideration is lightly damped and only the Young's modulus of an equivalent, homogeneous plate is of interest. The actual vibrational field $w(x, \omega)$ is approximated by $\phi_{k}(x)$, as follows

$$
\tilde{w}(x, \omega, k)=\alpha(k, \omega) \phi_{k}(x)
$$

where $\alpha_{k}$ is a frequency dependent scalar containing the contribution strength of the vector $\phi_{k}$ to the projected vibrational field $\tilde{w}$. Writing $w(x, \omega)$ and $\phi_{k}(x)$ as a vector $\mathbf{w}(\omega)$ and $\boldsymbol{\Phi}_{\mathbf{k}}$, respectively, the least squares solution for $\alpha$ is given by

$$
\alpha(k, \omega)=\boldsymbol{\Phi}_{\mathbf{k}}{ }^{+} \mathbf{w}(\omega)=\left(\boldsymbol{\Phi}_{\mathbf{k}}{ }^{*} \boldsymbol{\Phi}_{\mathbf{k}}\right)^{-1} \boldsymbol{\Phi}_{\mathbf{k}}{ }^{*} \mathbf{w}(\omega)
$$

where $\boldsymbol{\Phi}_{\mathbf{k}}{ }^{+}$is the pseudo inverse of $\boldsymbol{\Phi}_{\mathbf{k}}$. The error $e$ of the least squares fit can be defined as

$$
e(k, \omega)=\frac{\|\mathbf{w}(\omega)-\tilde{\mathbf{w}}(\omega, k)\|^{2}}{\|\mathbf{w}(\omega)\|^{2}},
$$

where $\tilde{\mathbf{w}}(\omega, k)$ is the vector representation of $\tilde{w}(x, \omega, k)$. This error $e$ obviously is a function of the assumed testing wavenumber $k$. Searching for the minimum of $e$ as function of the testing wavenumber $k$, an optimum value of $k$ can be found for each angular frequency $\omega$.

The wave fitting procedure, in addition, also allows the accuracy of the fit to be estimated. The confidence in the least squares fit results can be estimated as follows. Let's assume that each data point, as measured by the laser Doppler vibrometer, is drawn from a Gaussian distribution. Defining a 
reduced (i.e. corrected with a factor $1 /(N-P)$ ) goodness-of-fit parameter $\chi^{2}$ :

$$
\chi^{2}=\frac{1}{N-P} \sum_{i=1}^{N}\left\{\frac{\left[w\left(x_{i}\right)-\tilde{w}\left(x_{i}\right)\right]^{2}}{\sigma_{i}^{2}}\right\}
$$

where $w\left(x_{i}\right)$ is the measured data at measurement point $x=x_{i}$, having a standard deviation $\sigma_{i}, \tilde{w}\left(x_{i}\right)$ is the fitted data at that point, $N$ is the number of measurement data points and $P$ is the number of fit parameters (here: $P=1)$. In case the fit is correctly describing the phenomena, without systematic errors, it can be expected that the deviations $w\left(x_{i}\right)-\tilde{w}\left(x_{i}\right)$ are of the order of the expected standard deviations $\sigma_{i}$, causing the reduced goodnessof-fit parameter $\chi^{2}$ to approach unity for an optimum fit. Considering $\chi^{2}$ in terms of the (in this specific case a single) fitting variable $\alpha(k)$, it can be shown that near the vicinity of the minimum, $\chi^{2}$ can be approximated to second order as [27]

$$
\chi^{2}=\frac{\left(\alpha(k)-\alpha\left(k_{\text {opt }}\right)\right)^{2}}{(N-P) \sigma_{\alpha}^{2}}+1
$$

where $\alpha\left(k_{\text {opt }}\right)$ is the optimum value of for $\alpha(k)$ that minimizes $\chi^{2}$ and $\sigma_{\alpha}$ is the standard deviation of the fitting variable. Thus $\chi^{2}$ varies as the square of the distance from a minimum (which is equal to 1 for an unbiased fit), and an increase of 1 standard deviation $\left(\sigma_{\alpha}\right)$ in the parameter from the value $\alpha\left(k_{\text {opt }}\right)$ at the minimum increases $\chi^{2}$ by 1 [27]. Thus, the uncertainty in the $\alpha\left(k_{\text {opt }}\right)$, $\sigma_{\alpha}$, can be determined by plotting the error function $e$ (Eq. 7) as function of this fitting parameter, and search for the values of $a_{k}$ for which $e$ is increased by a factor 2 relative to the minimum of $e$. The standard deviation $\sigma_{\alpha}$ can be obtained by dividing this range by $\sqrt{N-P}$.

Once an estimate of the wavenumber $k$ as function of angular frequency is found, the stiffness of the plate can be estimated. To estimate the stiffness of a plate, a theoretical model is required to allow a conversion from wavenumber to the Young's modulus for bending. As the wavelength of the bendingwaves for the types of plates and frequencies considered in this paper is much larger than the thickness of the plate, the bending stiffness of the constrained-layer damping sandwich structure can be described by an equivalent homogeneous plate. The (frequency dependent) Young's modulus of such an equivalent, homogeneous thin plate following Kirchhoff-Love plate theory is seeked for. Considering Kirchhoff's thin shell theory, the following 
relation between the wavenumber $k$ and the Young's modulus $E$ exists:

$$
k=\sqrt[4]{\left(\frac{\omega^{2} m^{\prime}}{E I}\right)}
$$

Here $I$ is the second moment of area per unit width, and $m^{\prime}$ the mass per unit area, defined as

$$
m^{\prime}=\rho h
$$

where $\rho$ is the mass density and $h$ is the plate thickness. For a plate the second moment of area per unit width $I$ is given by

$$
I=\frac{h^{3}}{12\left(1-\nu^{2}\right)}
$$

where $\nu$ is Poisson's ratio. Using these relationships, the factor $E I / \mathrm{m}^{\prime}$ can be estimated from

$$
\frac{E I}{m^{\prime}}=\frac{\omega^{2}}{k^{4}}
$$

The advantage of considering only a limited number of waves propagating through the structure, as is done in Prony or in the wave fitting procedure, is that the wavenumber resolution is highly improved as compared to the classical spatial Fourier transform. However, the choice of the wave types must be an appropriate choice for this method to work well. For instance, when fitting the measurement data with only one flexural wave with wavenumber $k$, it is assumed that the higher order modes are negligible. It is assumed that the wave form consists of a single frequency component. For bending waves traveling in thin plates (relative to the wavelength), this assumption is usually justified. Application of the three fitting approaches will be given in the next sections.

\section{Measurement set up}

The reconstruction of dispersion curves requires the response of the structure (with a fixed point of excitation) to be measured at a number of points. Alternatively, using reciprocity, the structure can be excited at a number of points and the response of the structure is measured at a fixed point. It is the latter option that is used in this work, as it has the advantage that a retroreflecting sticker at a single point on the plate can be used to enlarge the 


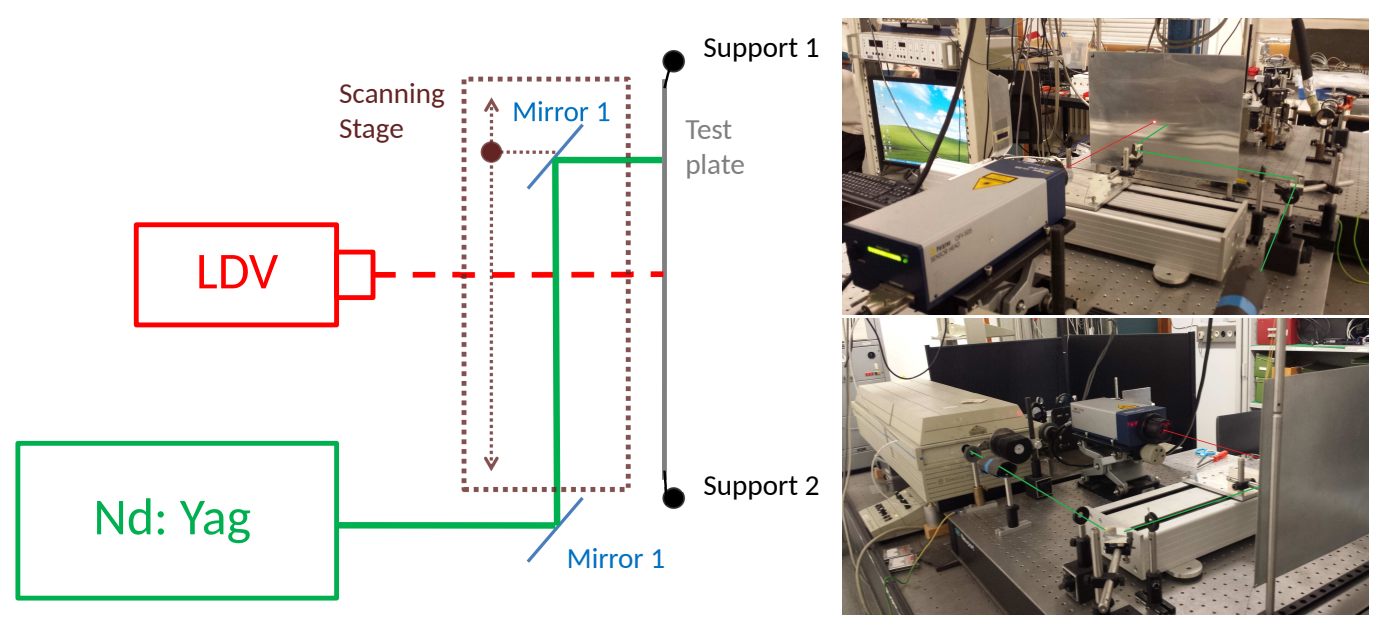

Figure 1: Measurement set up. Nd:YAG-laser beam illustrated by means of a green line. Laser Doppler Vibometer beam illustrated by means of a red line.

amount of laser light being reflected back into the laser Doppler vibrometer, thus improving the quality of the signal. Whilst the laser Doppler vibrometer measurement position is fixed, the Nd:YAG laser beam is moved across the plate.

Figure 1 shows the measurement set-up. The optical path of the Nd:YAGlaser beam (a Photonics diode pumped solid state powerchip nanolaser system, laser wavelength $1064 \mathrm{~nm}$, maximum energy $200 \mathrm{~mJ}$ per pulse, 10 pulses per second) is illustrated by means of a solid green line. The Nd:YAG-laser, however, was not used at its full power. The energy per pulse was reduced to about $10 \%$ in order not to cause permanent damage of the surface. The Nd:YAG-laser beam is moved across the plate from left to right by means of a home made translation stage.

The optical path of the Polytec Laser Doppler Vibrometer (Polytec laser head OFV-505 and a Polytec controller OFV-5000) is illustrated with of a dashed red line in Fig. 1. The Laser Doppler Vibrometer (LDV) was focussed on a fixed position of the plate, approximately at the middle of the scanning line.

Per measurement position a time record of $0.1 \mathrm{~s}$ was acquired, using a sample frequency of $f_{s}=500 \mathrm{kHz}$ (number of samples per time record $n_{t}=50001$ ). Time averaging was employed to improve the signal-to-noise ratio of the LDV signal. The firing moment of the Nd:YAG-laser was used to trigger the time- 
averaging process. A total of 500 time averages were taken per measurement position (thus requiring $500 \times 0.1=50 \mathrm{~s}$ measurement time per measurement position). The application of the time averaging is one of the reasons of having a high signal to noise ratio, as will be shown in Section 4. The plate response was measured at a fixed position by the LDV, as explained above, whilst the point of excitation was scanned along a line on the plate that coincides with the measurement point. The total scanning length is $0.381 \mathrm{~m}$, in steps of $1.5 \mathrm{~mm}$ (number of measurement positions $n_{x}=255$ ). Thus a matrix $w(x, t)$ with measurement data was obtained as function of $x$ and $t$, with a dimension of $n_{x} \mathrm{x} n_{t}=255 \times 50001$, with $x$ the coordinate of each point of excitation. As the light pulse of the Nd:YAG-laser is in the order of $1 \mathrm{~ns}$, all eigenfrequencies of the plate will be excited up to the $\mathrm{GHz}$ frequency range (although the measurable response of the plate is limited to about $50 \mathrm{kHz}$, due to inertia effects of the plate and the limited excitation force of the Nd:YAG laser pulses). As a result, the measurements contained in $w(x, t)$ can be considered to be an (unscaled) impulse response function of the plate.

The measurements were conducted on a constrained-layer damping sandwich plate, consisting of a thin steel layer of $0.30 \mathrm{~mm}$ thickness, a polymer layer of $0.69 \mathrm{~mm}$ thickness and a thin aluminum layer of $0.18 \mathrm{~mm}$ thickness (total plate thickness $h=1.17 \mathrm{~mm}$ ). The overall dimensions of the plate are $30 \mathrm{x} 40 \mathrm{~cm}^{2}$. The total measured weight of the plate was $0.387 \mathrm{~kg}$, yielding an effective mass per unit area $m^{\prime}=3.2 \mathrm{~kg} / \mathrm{m}^{2}$. The plate was suspended by means of elastic bands, thus realizing a 'free-free' boundary condition. With this type of boundary condition, the influence of the boundary conditions on the stiffness and damping of the plate is negligible.

\section{Measurement results and data processing results}

Figure 2 shows the measured impulse responses in $\mathrm{dB}$, computed as 20 . $\left.\log _{10}(|w(x, t)|)\right)$. Figure 3 shows the same data zoomed to early times $2<t<4$ ms. The fixed position of the laser Doppler vibrometer, denoted by $x=x_{0}$, was half way the plate, at $x_{0}=0.1973 \mathrm{~m}$. At the moment the Nd:YAG laser beam was hitting the plate (at approximately $t=2.2 \mathrm{~ms}$ ), the waves in the plate indeed seem to originate from $x=x_{0}$. Reflections from the ends of the plate at $x=0$ and $x=0.4 \mathrm{~m}$ started to occur at approximately $t=2.5 \mathrm{~ms}$. Between $2.2 \mathrm{~ms}<t<3 \mathrm{~ms}$ dispersion effects can be observed. 


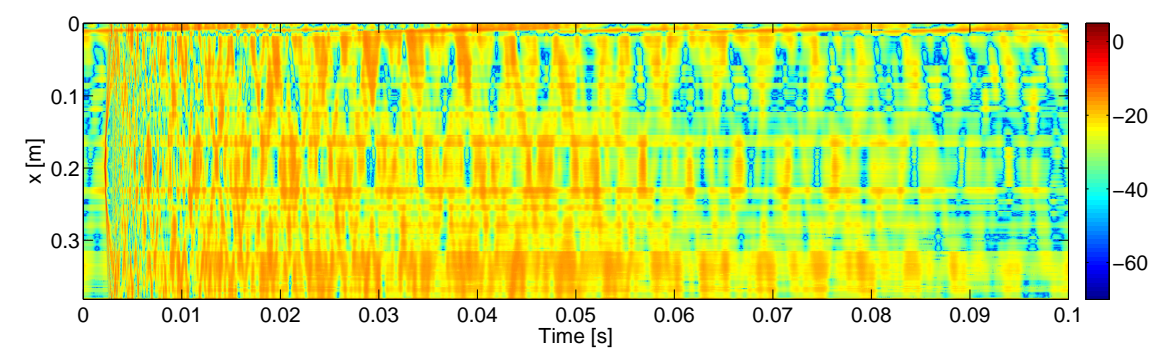

Figure 2: Dispersion measurement results as function of position $x$ and time $t$ (colorbar in $\mathrm{dB})$.

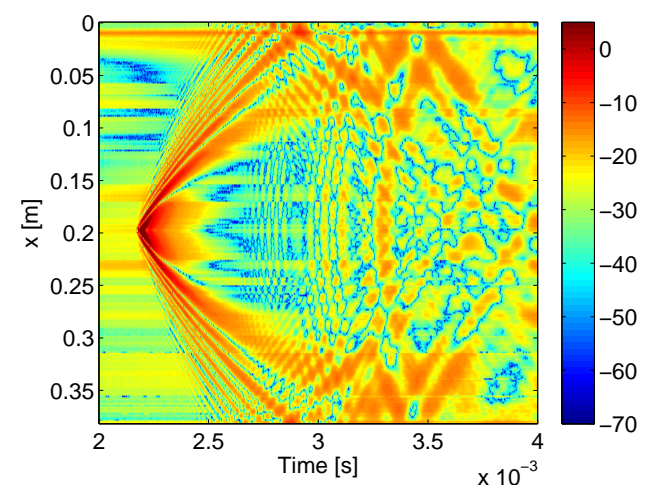

Figure 3: Dispersion measurement results as function of position $x$ and time $t$ (colorbar in $\mathrm{dB}$ ), as shown in Fig. 2. Time axis zoomed in on $2<t<4 \mathrm{~ms}$.

\subsection{Spatial Fourier transform results}

The measurement data $w(x, t)$ is transformed to the frequency domain by means of a fast Fourier transform (Eq. 11), to arrive at $w(x, \omega)$. No timedomain weighting function (such as Hanning for instance) is applied in this specific case to minimize leakage, as the signal is effectively reduced to the background level at the end of the time record (cfr. Fig. 2). The frequency $f=\omega / 2 \pi$ covers the range from $-f_{s} / 2$ up to $f_{s} / 2$ in $n_{t}$ steps, where $f_{s}$ is the sampling frequency of the time record and $n_{t}$ is the number of samples of the time record.

Subsequently, this data set is transferred from the spatial domain to the wavenumber domain, by means of a spatial fast Fourier transform (Eq. 2), 


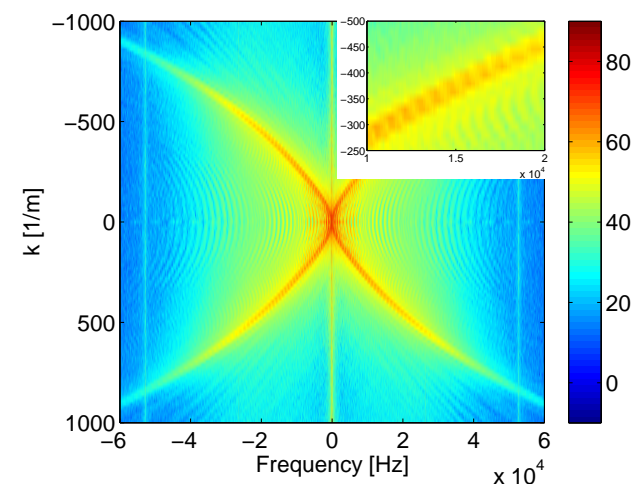

a)

b)

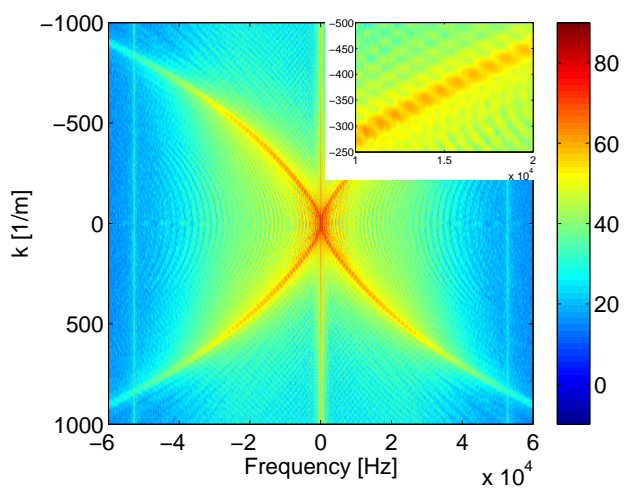

Figure 4: Dispersion measurement results as function of wavenumber $k$ and frequency $f$ (colorbar show dB's). a) No zero-padding. b) Zero-padding factor $=4$ for transformation from $x$ to $k$.

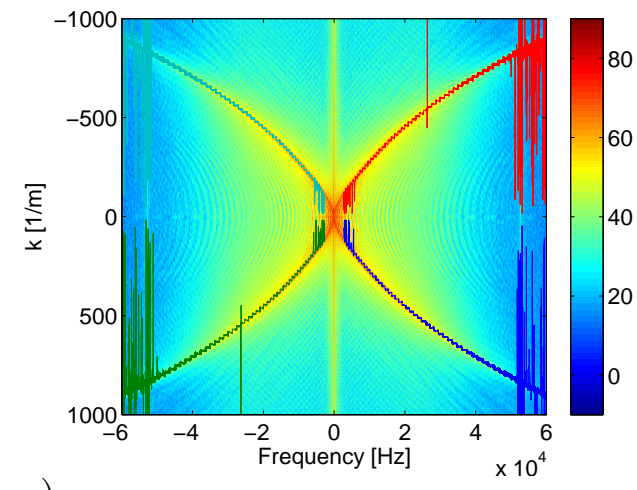

a)

b)

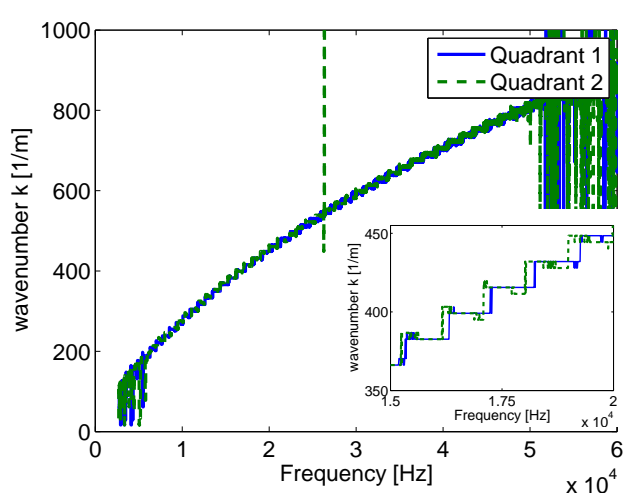

$$
\text { ) }
$$

Figure 5: Dispersion measurement results as function of wavenumber $k$ and frequency $f$ (zero-padding factor $=4$ for transformation from $x$ to $k$ ). The curves indicate the relationship between frequency and wavenumber, based upon maximum search of the spatial Fourier spectra data.

to arrive at a data set $w(k, \omega)$. Here the wavenumber $k$ is defined as $k=2 \pi / \lambda$, where $\lambda$ is the structural wavelength. The wavenumber ranges from $-k_{\max } / 2$ up to $k_{\max } / 2$ in $n_{x}$ steps, where $k_{\max }$ is the sampling frequency in the spatial domain and $n_{x}$ is the number of samples in the spatial domain. Here $k_{\max }$ is defined as $k_{\max }=2 \pi / d x$, where $d x$ is the spatial step size. 
Due to a limited scanning length of the plate, the wavenumber domain resolution, defined as $\Delta k=2 \pi / L$, where $L$ is the scanning length, was rather limited compared to the frequency domain resolution. In this specific case $(L=0.4 \mathrm{~m}), \Delta k=16 \mathrm{rad} / \mathrm{m}$. By means of zero-padding [28, 29] in the spatial domain (zero-padding factor $=4$ ), before taking the spatial Fourier transform, the resolution in the wavenumber domain was improved. The results can be seen in Fig. 4 for the case without zero-padding in the spatial domain, and with zero-padding. The effect of zero-padding improves the wave number domain resolution only slightly, as can be seen from the zooming-in overlay graphs.

Furthermore, the results shown in Fig. 4 show only one maximum in each quadrant, which clearly indicates that for the present set-up we are dealing with only one single wave propagating through the structure. Given the fact that the plate is excited in normal direction (i.e. an asymmetric excitation across the thickness of the plate), and the much higher flexibility of the plate for bending (antisymmetric A0 Lamb wave) as compared to compression (symmetric S0 Lamb wave), it is very likely that this single wave is a bending wave. The compression waves (symmetric S0 Lamb wave) remains below the noise level in Fig. 4.

Figure 5 shows the lines that indicate the maxima in the four quadrants of the $k-\omega$ domain, as obtained by the spatial Fourier transform (using zeropadding factor $=4$ for transformation from $x$ to $k$ ). Because the Fourier transform from the timedomain to the frequency domain is done on real signals, the results in the quadrants on the left and on the right of $f=0$ exhibit complex symmetry (being their complex conjugate). Thus, only the results of quadrant 1 and 2 are shown in Fig. 5b. This figure illustrates the earlier derived wavenumber resolution $\Delta k=16 \mathrm{rad} / \mathrm{m}$. Even when using zero-padding, the wavenumber resolution effectively remains the same.

\subsection{Prony fit results}

A Prony fit is pursued using two roots, one for each wave traveling away from the point of excitation, i.e. the waves running in positive and negative $x$-direction, respectively.

In addition to this two-pole Prony fit of the data, a Prony fit is pursued in which only one pole is used, employing only half of the measurement data set, i.e. for $x>x_{0}$, where $x_{0}=0.1973 \mathrm{~m}$ is the measurement position

of the laser Doppler vibrometer. Figure 6a shows the wavenumber $k$ as function of frequency $f$, based upon the measurement data for $x>x_{0}$, using 


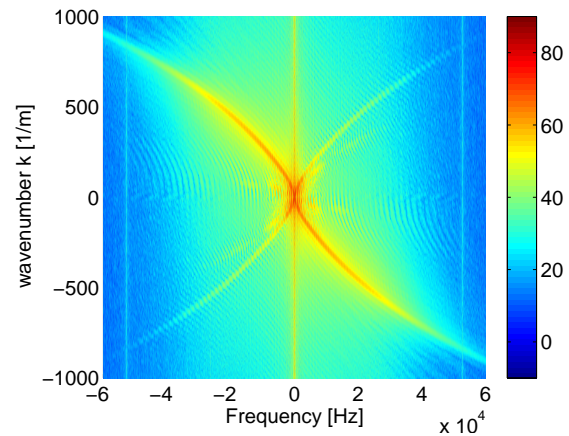

a)

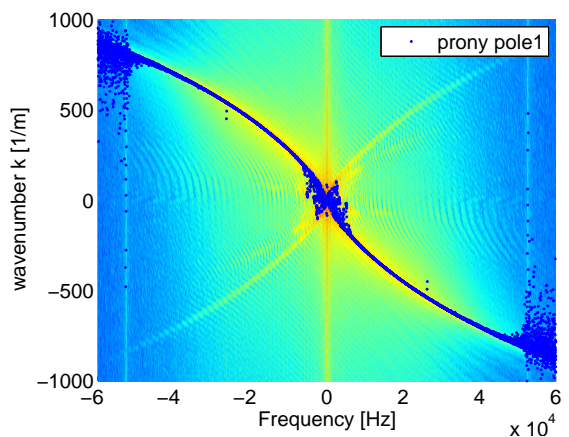

b)

Figure 6: a) Dispersion measurement results as function of wavenumber $k$ and frequency $f$, using measurement data for $x>x_{0}$ only. b) Prony results shown as blue dots.

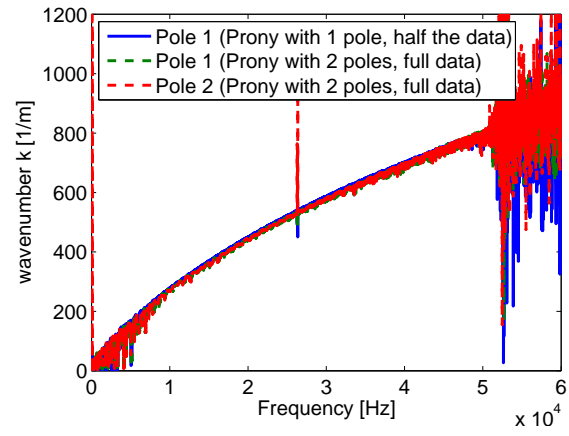

a)

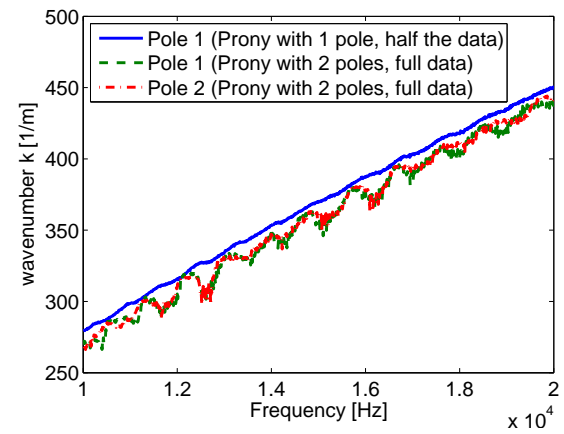

b)

Figure 7: Wavenumber $k$ estimate as function of frequency $f$, based upon Prony fit using one pole (using half the dataset) and based upon Prony fit with 2 poles (using the full dataset). a) Full frequency range. b) Zooming in on the frequency range from 10 to $20 \mathrm{kHz}$

a two-dimensional Fourier transform. Obviously, now only two of the four quadrants show high levels of vibration. Figure $6 \mathrm{~b}$ shows the Prony fit results.

It appears that the accuracy of the wavenumber estimate is improved when using only half the space-time dataset, employing a single-pole Prony fit. Figure 7 shows that the wavenumber estimate is much more smooth (which is physcially speaking more logical) for the single-pole Prony fit using half the space-time dataset as compared to the two-pole Prony fit using the complete space-time dataset. This effect is due to the disturbing influence 
of the measurement data on the left of the excitation point when fitting the right running waves, and vice versa. For this reason it is better to consider only half of the measurement data, either to the left or to the right of the point of excitation, and fit the data with a single Prony pole.

\subsection{Wave fitting results}

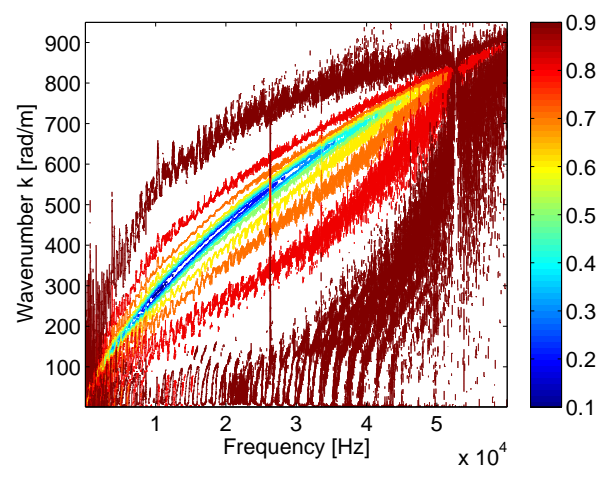

a)

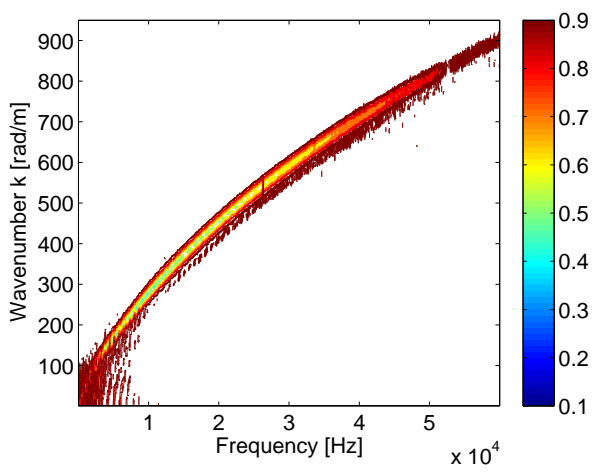

b)

Figure 8: Contour plots of the normalized error $e$ as function of testing wavenumber $k$ and frequency $f$. a) Using a test-wave $\phi_{k}=\mathrm{e}^{i k x} / \sqrt{\left|x-x_{0}\right|}$. b) Using a test-wave $\phi_{k}=e^{i k x}$.

Employing the wave fitting approach, Fig. 8 shows the normalized error $e$ (Eq. 7) as function of the fitting parameter $k$ and as function of frequency. In this specific case $k$ is considered to be real, as the damping of the plate is low. Fig. 8 a shows the error for the right running wave fit, using the measurement data for which $x>x_{0}$ and using a test-wave $\phi_{k}=e^{i k x} / \sqrt{\left|x-x_{0}\right|}$ (Eq. 4). Similar results are obtained for the left running wave fit (using the measurement data for which $x<x_{0}$ ). The resulting estimate of the wavenumber is shown in Fig. 9.

For purpose of illustration, Fig. 8b shows the error when using a test-wave $\phi_{k}=\mathrm{e}^{i k x}$. It is clear that the omission of the term $1 / \sqrt{\left|x-x_{0}\right|}$ that accounts for the geometric attenuation of the structural vibration level as function of the distance from the point of excitation, $x=x_{0}$, causes a higher normalized error $e$. Omitting the attenuation term results in a minimum error (best fit) of about 0.5 (Fig. 8b), whilst including the attenuation term results in a minimum error (best fit) of less than 0.1 (Fig. 8a). Clearly the attenuation term $1 / \sqrt{\left|x-x_{0}\right|}$ should be taken into account. 


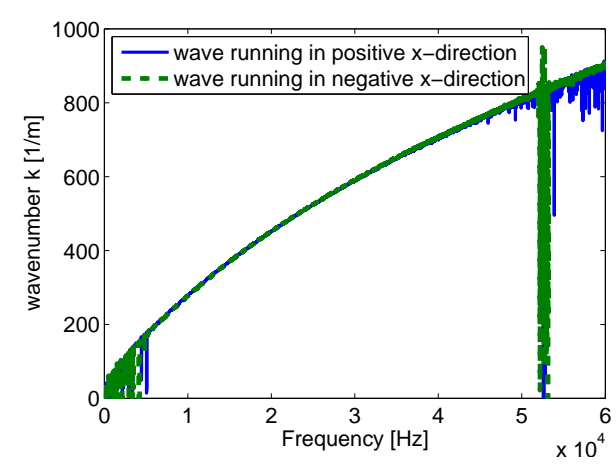

a)

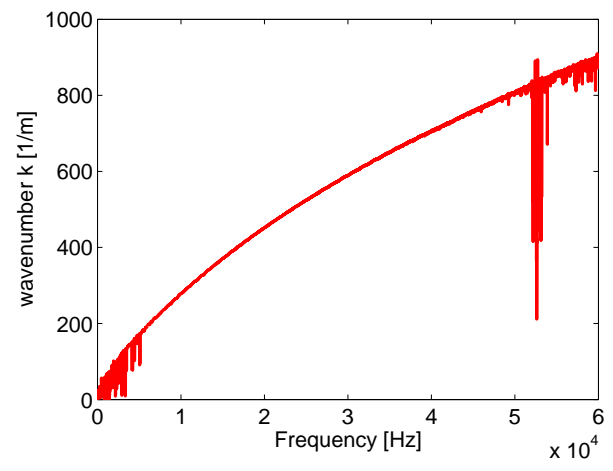

b)

Figure 9: Wavenumber $k$ estimate as function of frequency $f$, based upon wave fitting. a) using two waves running in positive and negative $x$-direction. b) using the mean of waves running in positive and negative $x$-direction.

In Fig. 10a the normalized error $e$ is shown for a frequency of $17.5 \mathrm{kHz}$, using both mentioned test-waves. Circles indicate the point where the error is twice the minimum error. The standard deviation $\sigma_{k}$ can be estimated following the procedure mentioned in Section 2 (just below Eq. 9p. The standard deviation $\sigma_{k}$, as function of frequency, can be obtained by searching for the wavenumber-shift relative to the optimum value for the wavenumber, for which the error is twice the minimum, and divide this wavenumber-shift by $\sqrt{N-P}$. In the present case the number of measurement data points $N$ is equal to 255 , and the number of fit parameters $P$ is equal to 1 . The resulting standard deviation $\sigma_{k}$ is presented in Fig. 10b. It shows that, when using the test wave $\phi_{k}=e^{i k x} / \sqrt{\left|x-x_{0}\right|}$ (Eq. 4), the standard deviation is in the order of $1 \mathrm{rad} / \mathrm{m}$ in the frequency range from $10 \mathrm{kHz}$ up to $30 \mathrm{kHz}$. The normalized standard deviation, i.e. $\sigma_{k} / k$, can be as low as $2 \cdot 10^{-3}$. This low value of the standard deviation is caused by the redundancy of measurements points $(N=255)$ and the high signal-to-noise ratio of the measurement when using time averaging.

As compared to the classical Fourier transform (wavenumber resolution $16 \mathrm{rad} / \mathrm{m}$ in the present case), it can be seen that the wavenumber resolution is improved by about a factor 15 . This is caused by the fact that the wavenumber fit (and the Prony fit) scan over all wavenumbers, whilst the wavenumber domain resolution of the classical Fourier transform is funda- 


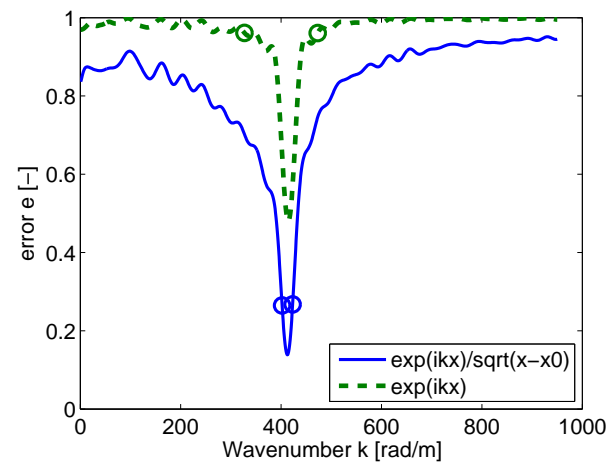

a)

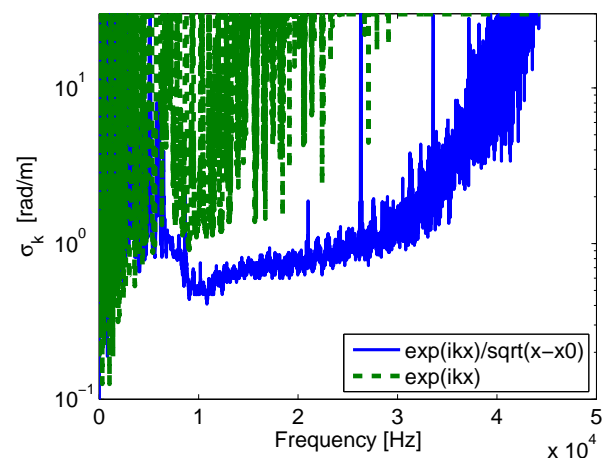

b)

Figure 10: Normalized error $e$ and standard deviation $\sigma_{k}$ using a test-wave $\phi_{k}=$ $e^{i k x} / \sqrt{\left|x-x_{0}\right|}$ (solid blue curves) and using a test-wave $\phi_{k}=e^{i k x}$ (dashed green curves). a) Normalized error $e$ as function of the wavenumber $k$ for a frequency of $17.5 \mathrm{kHz}$. b) Standard deviation $\sigma_{k}$ as function of frequency $f$.

mentally limited to $\Delta k=2 \pi / L$, where $L$ is the scanning length.

It should be noted that the application of the wavenumber fit (and the Prony fit) it is implicitely assumed that only a limited number of waves are present. For instance, in the wavenumber fit treated in this section, it is assumed there is only one single wave propagating in the plate. Obviously, this presumption must be valid. However, when this presumption is valid, a highly improved wavenumber resolution is obtained.

It should also be noted that it is required that the excitation point should be on the line of measurements in order to obtain a valid wavenumber estimate. If this would not be the case, a projection of the wavenumber on the measurement line will be obtained, which will give a bias deviation in comparison with the true wavenumber.

\subsection{Estimate of Young's modulus and comparison with an analytical model}

This section the Young's modulus of the constrained-layer damping sandwich plate under investigation is assessed and compared with an analytical model.

Considering the results of the previous sections, the wavenumber of the wave that was measured at a frequency of $50 \mathrm{kHz}$ is approximately equal to $800 \mathrm{rad} / \mathrm{m}$ (cfr. Figure 4, 6, 9). As the plate is excited in normal direction, the waves that are mainly excited are bending waves in the plate. Other 
waves are below the noise level of the measurement. The wavelength of these bending waves at a frequency of $50 \mathrm{kHz}$ is apparently approximately $\lambda=2 \pi / k=8 \mathrm{~mm}$, which is still large as compared to the thickness of the plate $(1.17 \mathrm{~mm})$. Thus, up to a frequency of $50 \mathrm{kHz}$, it is allowed to use the Kirchhoff-Love theory to estimate the Young's modulus of an equivalent, homogeneous plate. The Young's modulus can be computed by means of Eq. 13. assuming the material properties as mentioned in Section $3(h=1.17 \mathrm{~mm}$, $m^{\prime}=3.2 \mathrm{~kg} / \mathrm{m}^{2}$ ), and an assumed Poisson's ratio $\nu=0.33$.

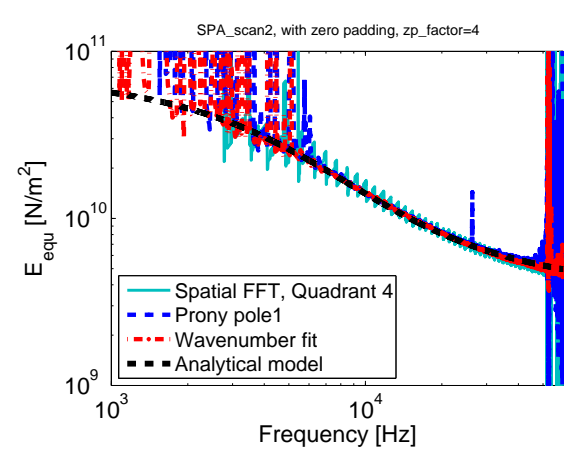

a)

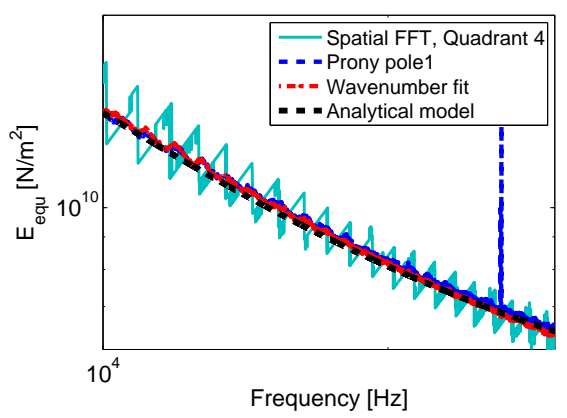

b)

Figure 11: Young's modulus $E_{\text {equ }}$ as function of frequency $f$, based upon maximum search in the spatial Fourier spectra data in quadrant 4 (solid magenta line), Prony fit (dashed blue line), and real wavenumber fit (dash-dotted red line). Analytical equivalent model in dashed thick black line. a) Full frequency range. b) Frequency range from 10 to $30 \mathrm{kHz}$.

Figure 11 combines the result from the three approaches in one figure. It shows the Young's modulus $E$ as function of frequency $f$ of an equivalent thin plate following Kirchoff's theory, based upon maximum search in the spatial Fourier spectra data, Prony fit and real wavenumber fit.

This figure also shows the equivalent Young's modulus of an equivalent homogeneous plate of same thickness and mass per unit area, that would exhibit the same dynamic flexural rigidity at one given frequency. This equivalent Youngs modulus is computed by the analytical model that was published by Guyader [30, 31]. The analytical method is based on the travelling wave approach applied to a simplified multi-layer model. In each layer bending, membrane and shear effects are considered. Continuity conditions on displacement and shear stresses at each layer interface are used to obtain the equations of motion of the multi-layered plate field expressed in the 


\begin{tabular}{l||l|l|l|} 
& layer 1 & layer 2 & layer 3 \\
\hline Thickness $h[\mathrm{~m}]$ & $0.3 \times 10^{-3}$ & $0.69 \times 10^{-3}$ & $0.18 \times 10^{-3}$ \\
Young's modulus $E\left[\mathrm{~N} / \mathrm{m}^{2}\right]$ & $210 \times 10^{9}$ & $300 \times 10^{6}$ & $69 \times 10^{9}$ \\
Density $\rho\left[\mathrm{kg} / \mathrm{m}^{3}\right]$ & 7800 & 580 & 2700 \\
Poisson's ratio $\nu[-]$ & 0.33 & 0.33 & 0.33
\end{tabular}

Table 1: Dimensions and material properties of the individual layers of the constrainedlayer damping sandwich plate, as assumed in the analytical model.

function of the first layer field. The input parameters for the computation of the analytical equivalent Young's modulus is in correspondence with the dimensions mentioned in Section 3, as summarized in Table 1. The material properties for aluminum and steel as known from the literature were used. The density of the plastic layer was measured, while the Young's modulus of this layer was fitted on the measurement data.

From Fig. 11 it can be seen that both the estimate of the Young's modulus which is based upon the Prony fit and the wavenumber fit are very smooth and compare very well with the analytically predicted equivalent Young's modulus. It can also be seen that the assessment of the wavenumber, and the resulting estimate for the frequency dependent equivalent Young's modulus $E_{\text {equ }}$, on the basis of the spatial Fourier transform has problems with the wavenumber resolution.

The estimated standard deviation $\sigma_{k}$ in the wavenumber estimate as obtained by the wave fitting approach of approximately $1 \mathrm{rad} / \mathrm{m}$ in the frequency range from $10 \mathrm{kHz}$ up to $30 \mathrm{kHz}$, and normalized standard deviation $\sigma_{k} / k$ is as low as $2 \cdot 10^{-3}$. Thus the normalized standard deviation of the Young's modulus, as a result of the $4^{\text {th }}$ power in Eq. 13 , equals approximately $4 \times 2 \cdot 10^{-3} \approx 1 \cdot 10^{-2}$. However, the estimate of the Young's modulus also depends on the assumed thickness, density and Poisson's ratio, which each also incorporate an uncertainty.

\section{Conclusions}

An all-optical, non-contact measurement approach for both excitation and response measurement was employed to assess the wavenumber and the dynamic stiffness of a constrained-layer damping sandwich plate. The measurements were taken along a line on the plate, thus reducing the measurement effort without compromising the quality of the wavenumber assessment. 
Repeatedly exciting the plate by a Nd:YAG laser beam, time averaging improved the signal-to-noise ratio of the measurements to a maximum of 100 $\mathrm{dB}$, without DOF-jitter effects.

Three methods for the estimation of the wavenumber as function of frequency were used; the spatial Fourier approach, the Prony approach and the wave fitting approach. Both the Prony approach and the wave fitting approach give a good wavenumber domain resolution. The estimates of both methods compare very well with the analytical results, confirming the correctness of the estimated equivalent Youngs modulus as function of frequency. Moreover, the wave fitting approach allows the accuracy of the wave number to be estimated, which is not possible with the other methods. Furthermore, it is shown that the spatial Fourier approach suffers from a limited wavenumber domain resolution, which is dictated by the scanning length.

The standard deviation of the wavenumber estimate as obtained by the wave fitting approach was in the order of $1 \mathrm{rad} / \mathrm{m}$ in the frequency range from $10 \mathrm{kHz}$ up to $30 \mathrm{kHz}$. The best normalized standard deviation was $2 \%$ for the wavenumber estimate and $4 \times 2=8 \%$ for the Young's modulus estimate. This low value of the standard deviation is caused by the redundancy of measurements points and the high signal-to-noise ratio of the measurement when using time averaging. The wavenumber domain resolution of the classical Fourier transform was $16 \mathrm{rad} / \mathrm{m}$ for the case studied.

\section{Acknowledgments}

This work was supported by the Labex CeLyA of Université de Lyon, operated by the French National Research Agency (ANR-10-LABX-0060/

ANR-11-IDEX-0007) and by INSA-Lyon (BQR VIVARIUM project). The authors thank Bas Möllenkramer for his suggestions concerning the manuscript. 
[1] P. J. Shorter, Wave propagation and damping in linear viscoelastic laminates J. Acoust. Soc. Am., Vol. 115, No. 5, Pt. 1, May 2004.

[2] Moreira, R. A. S., Rodrigues, J. D., and Ferreira, A. J. M. (2006). "A generalized layerwise finite element for multi-layer damping treatments," Computational Mechanics 37, 426-444.

[3] D. Chronopoulos, B. Troclet, O. Bareille, M. Ichchou, Modeling the response of composite panels by a dynamic stiffness approach, Composite Structures, Volume 96, February 2013, Pages 111-120, ISSN 0263-8223, http://dx.doi.org/10.1016/j.compstruct.2012.08.047.

[4] D. Chronopoulos, M. Ichchou, B. Troclet, O. Bareille, Efficient prediction of the response of layered shells by a dynamic stiffness approach, Composite Structures, Volume 97, March 2013, Pages 401-404, ISSN 0263-8223, http://dx.doi.org/10.1016/j.compstruct.2012.10.012.

[5] J. R. Banerjee, Dynamic stiffness formulation for structural elements: a general approach. Computers and Structures 63 (1) (1997) 101103.

[6] J.R. Banerjee, H. Su, C. Jayatunga, A dynamic stiffness element for free vibration analysis of composite beams and its application to aircraft wings. Computers and Structures 86 (2008) 573579

[7] E. Nilsson, A. C. Nilsson, Prediction and measurement of some dynamic properties of sandwich structures with honeycomb and foam cores, Journal of Sound and Vibration 251 (3) (2002) 409-430, 64.

[8] D. Backstrom, A. C. Nilsson, Modelling the vibration of sandwich beams using frequency-dependent parameters, Journal of Sound and Vibration $300(3-5)$ (2007) 589-611.

[9] S. Gade, H. Herlufsen, , a hand-held exciter for field mobility measurements - an alternative to the impact hammer method., in: IMAC-XI, 11th International Modal Analysis Conference - Modal Testing and Analysis, February 14, 1993 Hyatt Orlando Hotel, Kissimmee, Florida., Society for Experimental Mechanics, Inc., 1993.

[10] B. T. Hefner, P. L. Marston, Magnetic excitation and acoustical detection of torsional and quasi-flexural modes of spherical shells in water, Journal of the Acoustical Society of America 106 (6) (1999) 3340-3347. 
[11] S. Vanlanduit, F. Daerden, P. Guillaume, Experimental modal testing using pressurized air excitation, Journal of Sound and Vibration 299 (1-2) (2007) 83-98.

[12] M. Bau, V. Ferrari, D. Marioli, E. Sardini, M. Serpelloni, A. Taroni, Contactless electromagnetic excitation of resonant sensors made of conductive miniaturized structures, Sensors and Actuators a-Physical 148 (1) (2008) 44-50.

[13] R. Farshidi, D. Trieu, S. S. Park, T. Freiheit, Non-contact experimental modal analysis using air excitation and a microphone array, Measurement 43 (6) (2010) 755-765.

[14] P. Castellini, G. M. Revel, L. Scalise, Measurement of vibrational modal parameters using laser pulse excitation techniques, Measurement 35 (2) (2004) 163-179.

[15] S. L. Marple, Digital Spectral Analysis with Applications. Prentice-Hall 1987.

[16] S. Braun, Y. M. Ram, Determination of structural-modes via the prony model - system order and noise induced poles, Journal of the Acoustical Society of America 81 (5) (1987) 1447-1459.

[17] K. Grosh, E. G. Williams, Complex wave-number decomposition of structural vibrations, Journal of the Acoustical Society of America 93 (2) (1993) 836-848, grosh, Karl/0000-0001-8704-6199 22.

[18] J. Berthaut, M. N. Ichchou, L. Jezequel, K-space identification of apparent structural behaviour, Journal of Sound and Vibration 280 (35) (2005) 1125-1131, iCHCHOU, Mohamed/M-5352-2013 ICHCHOU, Mohamed/0000-0002-2452-0831 13.

[19] C. R. Halkyard, Maximum likelihood estimation of flexural wavenumbers in lightly damped plates, Journal of Sound and Vibration 300 (1-2) (2007) 217-240.

[20] M. Rak, M. Ichchou, J. Holnicki-Szulc, Identification of structural loss factor from spatially distributed measurements on beams with viscoelastic layer, Journal of Sound and Vibration 310 (4-5) (2008) 801-811. 
[21] P. J. Halliday, K. Grosh, Maximum likelihood estimation of structural wave components from noisy data, Journal of the Acoustical Society of America 111 (4) (2002) 1709-1717.

[22] L. Hillstrom, M. Mossberg, B. Lundberg, Identification of complex modulus from measured strains on an axially impacted bar using least squares, Journal of Sound and Vibration 230 (3) (2000) 689-707.

[23] Y. B. Liao, V. Wells, Estimation of complex modulus using wave coefficients, Journal of Sound and Vibration 295 (1-2) (2006) 165-193.

[24] A. J. Hull, D. A. Hurdis, A parameter estimation method for the flexural wave properties of a beam, Journal of Sound and Vibration 262 (1) (2003) 187-197.

[25] E. Ventsel, T. Krauthammer, Thin Plates and Shells: Theory: Analysis, and Applications, Marcel Dekker, Inc., New York, 2001.

[26] D. J. Trudnowski, J. M. Johnson, J. F. Hauer, Making prony analysis more accurate using multiple signals, Ieee Transactions on Power Systems 14 (1) (1999) 226-231.

[27] P. R. Bevington, D. K. Robinson, Data Reduction and Error Analysis for the Physical Sciences, McGrawHill, New York, 2003.

[28] T. Grandke, Interpolation algorithms for discrete fourier-transforms of weighted signals, Ieee Transactions on Instrumentation and Measurement 32 (2) (1983) 350-355.

[29] J. Schoukens, R. Pintelon, H. Vanhamme, The interpolated fast fouriertransform - a comparative-study, Ieee Transactions on Instrumentation and Measurement 41 (2) (1992) 226-232.

[30] J. L. Guyader, C. Lesueur, Acoustic transmission through orthotropic multilayered plates, part .1. plate vibration modes, Journal of Sound and Vibration 58 (1) (1978) 51-68, 18.

[31] J.-L. Guyader, C. Cacciolati, Viscoelastic properties of single layer plate material equivalent to multi-layer composites plate., in: INTER-NOISE and NOISE-CON Congress and Conference Proceedings, Vol. 2007 of 3, Institute of Noise Control Engineering, Institute of Noise Control Engineering, 2007. 